https://doi.org/10.15407/ujpe64.7.577

YU. GORNUSHKIN

on behalf of the DsTau Collaboration

Joint Institute for Nuclear Research

(6, Joliot-Curie Str., Dubna, 141980, Russia; e-mail: gornshk@yandex.ru)

\title{
STUDY OF TAU NEUTRINO PRODUCTION IN PROTON NUCLEUS INTERACTIONS
}

\begin{abstract}
In the DsTau experiment at the CERN SPS, an independent direct way to study the tau neutrino production in high energy proton-nucleous interactions was proposed. Since the main source of tau neutrinos is a decay of $D_{s}$ mesons, the project aims at measuring the differential cross-section of this reaction. The experimental method is based on the use of high-resolution emulsion detectors for the efficient registration of events with short-lived particle decays. The motivation of the project, details of the experimental technique, and the first results of the analysis of the data collected during test runs, which prove the feasibility of the study are presented.

Ke ywords: tau neutrino, cross-section, nuclear emulsions.
\end{abstract}

\section{Introduction}

Tau neutrino is eventually the least studied elementary particle. Although its existence was predicted after the tau lepton discovery in 1975 [1], the first tau neutrinos were detected in the DONuT experiment 25 years later [2]. In 2015, somewhat more $\nu_{\tau}$ appeared through $\nu_{\mu} \leftarrow \nu_{\tau}$ oscillations were detected by OPERA [3]. Super-Kamiokande (SK) and IceCube [4] also reported an evidence of the $\nu_{\tau}$ presence in their data.

Given a poor statistics of registered tau neutrinos, their properties are not well studied. In particular, the cross-section of the tau neutrino charge current (CC) interaction is known [5] with much larger statistical and systematic uncertainties compared to the other neutrino flavors, as shown in Fig. 1. However, a precise measurement of this cross-section would allow testing of the Lepton Flavor Universality (LFU) in the neutrino scattering. LFU is a principal assumption of the Standard Model (SM) of particle physics, but its validity was questioned by recent results on the B decay asymmetry [6-8]. There is the expectation of a possible deviation of the cross-section of the $\nu_{\tau}$ interaction as well [9]. The measurement of the $\nu_{\tau}$ CC cross-section has impact on the current and future neutrino oscillation experiments. In the mass hierarchy measurements in the atmospheric Super-

(c) YU. GORNUSHKIN, 2019

ISSN 2071-0194. Ukr. J. Phys. 2019. Vol. 64, No. 7
Kamiokande (SK) [10] and accelerator neutrino experiments (e.g., in DUNE [11] and HyperKamiokande $[12]), \nu_{e}$ flux measurement will have a background due to $\tau \rightarrow e$ decays. So, the systematic uncertainty of the $\nu_{\tau}$ interaction cross-section will be a limiting factor in the oscillation analyses in these experiments $[13,14]$.

So far, the tau neutrino interaction cross-section was only measured in the DONuT [5], OPERA [18], and SK [17] experiments, though under rather different conditions. All the measurements have large statistical and/or systematic errors of $30-50 \%$ due to low statistics and experimental uncertainties. In a future experiment at CERN, SHiP [19], a rich neutrino program [20] is proposed with thousands of tau neutrino interactions detected, hence, providing a negligible statistical error of the cross-section measurement. The overall accuracy of the cross-section will be determined by the systematic errors, and, in particular, by the $\nu_{\tau}$ flux uncertainty, which is to be studied by the DsTau experiment [34].

The dominant source $(>90 \%)$ of $\nu_{\tau}$ in an accelerator-based neutrino beam is leptonic decays of $D_{s}^{ \pm}$mesons produced in proton-nucleus interactions:

$D_{s}^{-} \rightarrow \tau^{-} \bar{\nu}_{\tau}$,

$\tau^{-} \rightarrow X \nu_{\tau}$

producing $\nu_{\tau}$ and $\bar{\nu}_{\tau}$ in every decay.

Conventionally, the differential production crosssection of charmed particles is approximated by a 

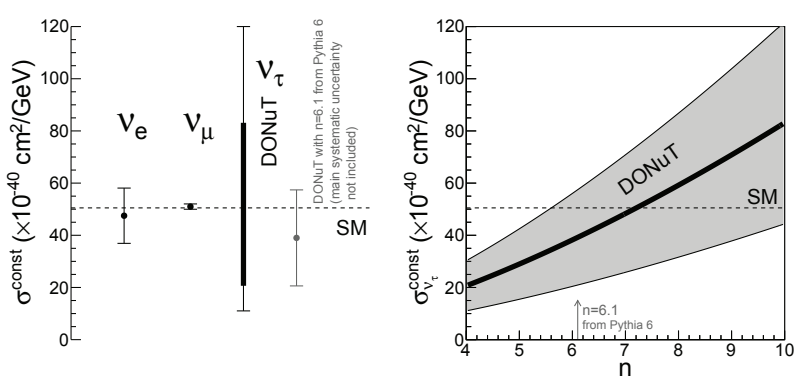

Fig. 1. Left: $\nu, \bar{\nu}$ averaged energy-independent cross-section of the three neutrino flavors ([15] for $\nu_{e},[16]$ for $\nu_{\mu}$, and for $\left.\nu_{\tau}[5]\right)$. The SM LFU prediction is indicated as a dashed horizontal line. For the DONuT result, since there is no measurement of the parameter $n$ concerning the $D_{s}$ double differential production cross-section (Eq. 1), the value is plotted in the empirical range of a parameter $n$ given by the DONuT paper as in the right plot

phenomenological formula

$\frac{d^{2} \sigma}{d x_{\mathrm{F}} \cdot d p_{T}^{2}} \propto\left(1-\left|x_{F}\right|\right)^{n} \cdot e^{-b \cdot p_{T}^{2}}$,

where $x_{\mathrm{F}}$ is the Feynman $x\left(x_{\mathrm{F}}=2 p_{Z}^{\mathrm{CM}} \sqrt{ } s\right)$ and $p_{T}$ is the transverse momentum, $n$ and $b$ are the parameters controlling the longitudinal and transverse dependences of the differential production cross-section, respectively. Although there were several measurements on charm particles [21-25], there is a lack of measurements on the $D_{s}$ differential production cross-section in the proton interactions, especially concerning the longitudinal dependence represented by the parameter $n$. This has been the main source of the systematic uncertainty of the $\nu_{\tau}$ cross-section measurements in DONuT [5].

Thus, a new measurement of the differential production cross-section of $D_{s}$ is necessary for future precise tau neutrino measurements, as well as for the reevaluation of the DONuT result. In the DsTau experiment, a direct study of the tau neutrino production, namely, the measurement of $D_{s} \rightarrow \tau \rightarrow X$ decays following high-energy proton-nucleus interactions, is proposed. DsTau will provide an independent $\nu_{\tau}$ flux prediction for future neutrino beams with accuracy under $10 \%$. Then the systematic uncertainty of the $\nu_{\tau} \mathrm{CC}$ cross-section measurement can be made sufficiently low to test LFU in the neutrino scattering by future experiments [20].

In addition to the primary aim of measuring the $D_{s}$ differential production cross-section in $2.3 \times 10^{8}$ pro- ton interactions, a high yield of $\mathcal{O}\left(10^{5}\right)$ charmed particle pairs is expected. The analysis of those events can provide valuable by-products.

\section{Overview of the Project}

DsTau exploits a simple setup consisting of a segmented high-resolution nuclear emulsion vertex detector (a module) capable to recognize $D_{s} \rightarrow \tau \rightarrow X$ by their very peculiar double-kink topology as shown in the bottom part of Fig. 2. In addition, because charm quarks are created in pairs, another decay of a charged/neutral charmed particle from the same vertex will be observed with a flight length of a few millimeters. Such a "double-kink plus decay" topology in a short distance has a marginal background.

However, to register the events is a challenge. First, all the decays take place on a scale of millimeters: the mean flight lengths of $D_{s}, \tau$, and pair-charms are 3.6, 2.1 , and $4.2 \mathrm{~mm}$, respectively. Second, although the kink angle at the $\tau$ decay vertex is easily recognizable (mean kink angle of $96 \mathrm{mrad}$ ), the one at $D_{s} \rightarrow \tau$ decays is rather small, $6.2 \mathrm{mrad}$. The expected signal features were studied making use of Pythia 8.1 [29]. The project aims to detect $\sim 1000 D_{s} \rightarrow \tau \rightarrow X$ decays in $2.3 \times 10^{8}$ proton interactions with a tungsten target. State-of-the-art nuclear emulsion detectors with a nanometric-precision readout will be used to achieve this goal. The modern use of the emulsion detection technology is based on the high-speed high-precision automatic readout of emulsions developed during the last two decades and available today [26-28].

The DsTau module structure is shown in Fig. 2. The upstream part is named the decay module. The basic unit is made of a $500 \mu \mathrm{m}$-thick tungsten plate (target) followed by 10 emulsion films interleaved with $9200 \mu \mathrm{m}$-thick plastic sheets which act as a decay volume for short-lived particles, as well as highprecision particle trackers. This structure (thickness of $5.4 \mathrm{~mm}$ ) is repeated 10 times. Five additional emulsion films are placed most upstream of the module to tag the incoming beam protons. It is followed by the downstream part made of a repeated structure of emulsion films and 1-mm-thick lead plates for the measurement of the momenta of daughter particles through their Multiple Coulomb Scattering (MCS) measurement [30]. The entire detector module is $12.5 \mathrm{~cm}$ wide, $10 \mathrm{~cm}$ high, and $8.6 \mathrm{~cm}$ thick and consists of a total of 131 emulsion films. 


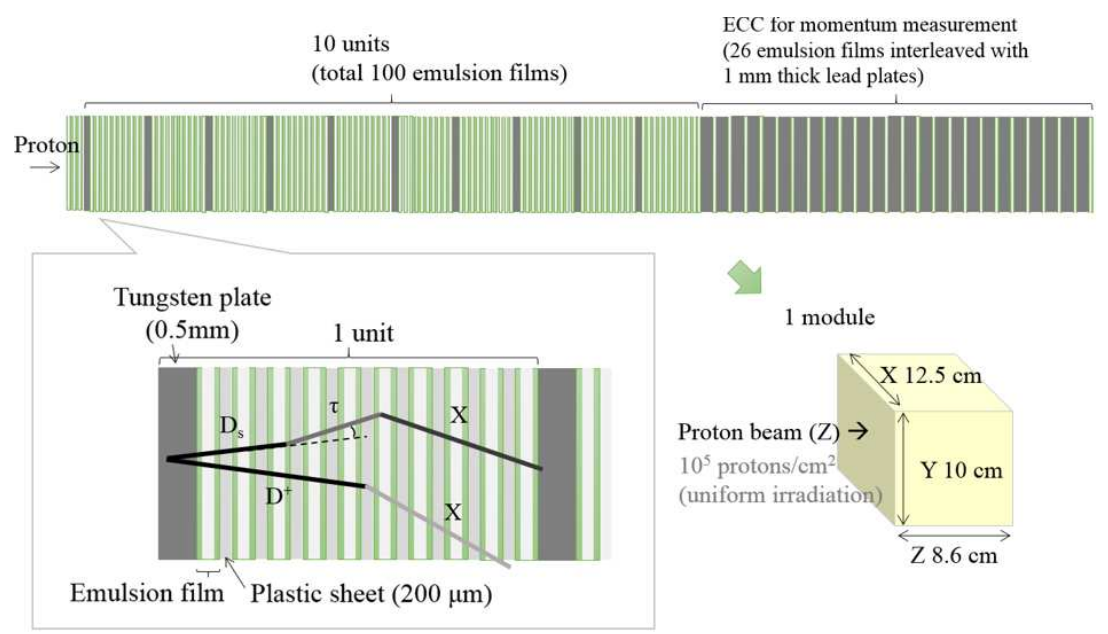

Fig. 2. Schematic view of the module structure. A tungsten target plate is followed by 10 emulsion films alternated by 9 plastic sheets acting as a tracker and a decay volume of $5.4 \mathrm{~mm}$. The sensitive layers of emulsion detectors are indicated by green color. This basic structure is repeated 10 times, and then followed by a lead-emulsion structure for the measurement of the momenta of daughter particles. In the bottom part, the "double kink" topology of $D_{s} \rightarrow \tau \rightarrow X$ is shown

Once a charged particle passes through the emulsion layer, the ionization is recorded quasipermanently and then amplified and fixed by the chemical process. The trajectory of a charged particle can be observed on an optical microscope. The emulsion detector with $200 \mathrm{~nm}$-diameter $\mathrm{AgBr}$ crystals and a 210 $\mu m$-thick base has a track position resolution of 50 $\mathrm{nm}$ [32] and an angular resolution of $0.34 \mathrm{mrad}$ (projection). With this angular resolution, one can detect 2 -mrad kink with $4 \sigma$ confidence.

A key feature of the modern emulsion technique is the use of fast readout instruments, which allow extracting and digitizing the information on the tracks fully automatically. Emulsion detectors and automated readout systems have been successfully employed in several neutrino experiments such as CHORUS [33], DONuT [2,5] and OPERA [3]. The latest scanning system, HTS [26,27], allows the scanning of emulsion films at a speed of $5,000 \mathrm{~cm}^{2}$ per hour per emulsion layer, which is $\mathcal{O}(100)$ faster than those used in OPERA.

The detection efficiency for the $D_{s} \rightarrow \tau \rightarrow 1$ prong events ( $85 \%$ of $\tau$ decays) was estimated by the PYTHIA 8.1 [29] simulation.

The following criteria were requested to be fulfilled: (1) the parent particle has to pass through at least one emulsion film (two sensitive layers), (2) the first kink daughter has to pass through at least two sensitive layers, and the kink angle is $\geq 2 \mathrm{mrad}$, (3) the flight length of the parent and the first kink daughter has to be $<5 \mathrm{~mm}$, (4) the second kink angle is $\geq 15 \mathrm{mrad}$ and (5) the partner of the charm pair is detected with $0.1 \mathrm{~mm} \leq$ flight length $<5 \mathrm{~mm}$ (they can be charged decays with a kink angle $>15$ mrad or neutral decays). With these selection criteria, the detection efficiency was estimated to be $20 \%$.

The main background to $D_{s} \rightarrow \tau \rightarrow 1$ prong events is hadron interactions that can mimic the decays of short-lived particles. Its probability was obtained by simulating $3 \times 10^{5}$ protons on the detector with the FLUKA [35] simulation. The criteria used for the charged charm or tau decay topology selection are applied to the interactions of secondary hadrons with only one charged daughter particle $(P>2 \mathrm{GeV} / \mathrm{c})$. In addition to high-energy particles, a large part of interactions has associated nuclear fragments, which is a strong evidence of hadron interactions. Those are effectively rejected by requesting only one charged daughter. The total probabilities to account for background events such as a double kink with charged pair charm or with neutral pair charm are $1.3 \pm 0.4 \times 10^{-9}$ and $2.7 \pm 0.8 \times 10^{-9}$ per incident proton, respectively. The expected numbers of background events in the full statistics of DsTau $\left(4.6 \times 10^{9}\right.$ p.o.t. $)$ are $6.0 \pm 1.8$ and $12.4 \pm 3.7$ for these 2 -signal channels, respectively.

DsTau will provide the differential cross-section of $D_{s}$ meson production and the following decay to a 


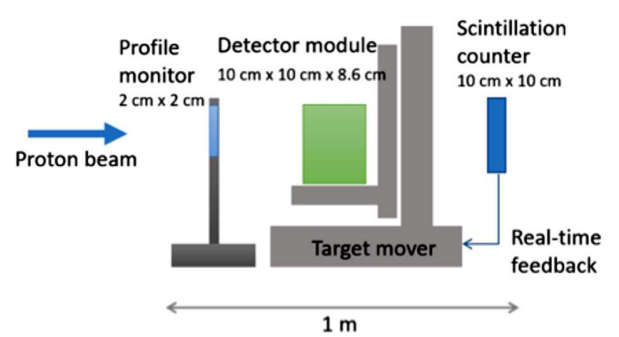

Fig. 3. Schematic of the DsTau setup. The detector module was moved in the plane perpendicular to the beam to provide uniform exposure at a density of $10^{5}$ protons $/ \mathrm{cm}^{2}$

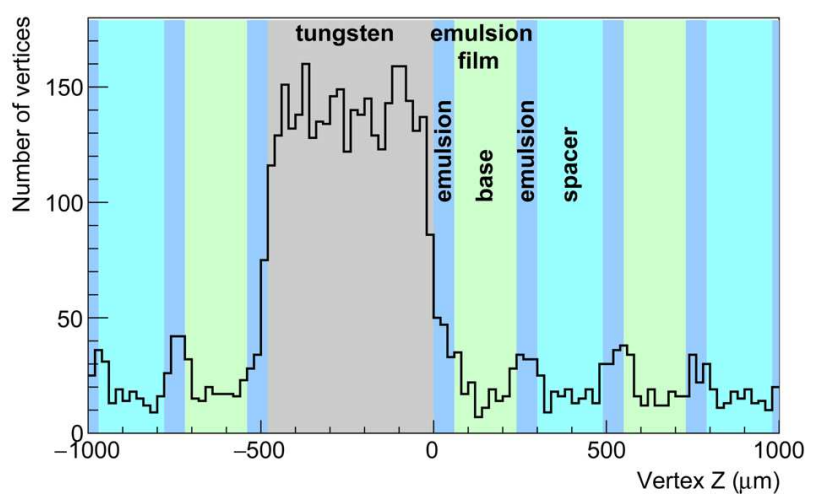

Fig. 4. Reconstructed vertex position distribution in $Z$. The correspondence with the detector structure is clearly visible

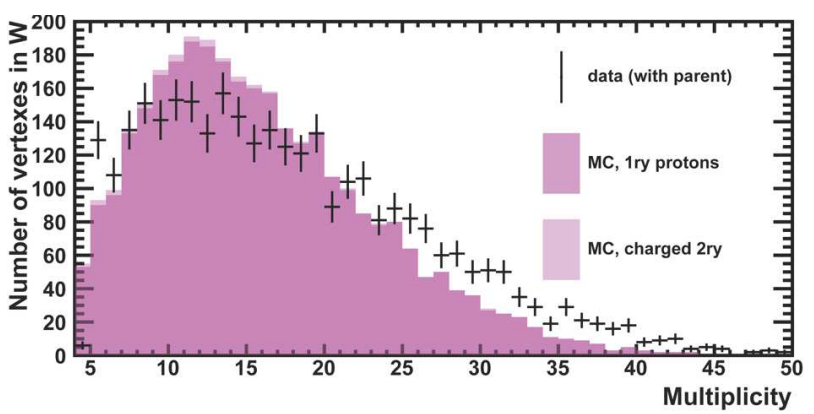

Fig. 5. Measured multiplicity of charged particles at the proton interaction vertices compared with the prediction from FLUKA simulations

tau lepton in the $400-\mathrm{GeV}$ proton-nucleus interaction. It may be fit with the phenomenological formula, Eq. (1), and get the parameter $n$ estimated, which is relevant for a re-evaluation of the tau neutrino cross-section measurement by the DONuT experiment. At the statistics of $1000 D_{s} \rightarrow \tau \rightarrow X$ detected events, the relative uncertainty of the $\nu_{\tau}$ flux will be reduced to below $10 \%$ [34].

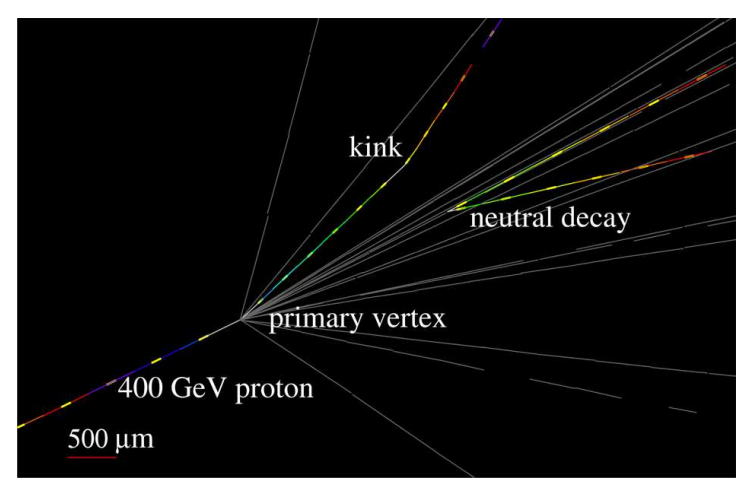

Fig. 6. A double charm candidate event with neutral 2-prong (vee) and charged 1-prong (kink) topologies. (tilted view) See the text about the details of event features

In order to collect $1000 D_{s} \rightarrow \tau$ events, 230 millions of proton interactions are to be analyzed, which is another challenge from the point of view of the track density and the amount of data to be processed. The high proton density of $10^{5} \mathrm{~cm}^{2}$ at the upstream surface of an emulsion detector was chosen to maximize the number of interactions in a single module. The track density will then increase in the detector, yet not exceeding $10^{6} \mathrm{~cm}^{2}$ at the downstream part of the decay module, which is affordable for the emulsion detector readout and reconstruction. With this density, $6.25 \times 10^{5}$ proton interactions are expected in the tungsten target in a decay module. To accumulate $2.3 \times 10^{8}$ proton interactions in the tungsten plates, $4.6 \times 10^{9}$ protons on the target are needed. About 370 modules with a total film area of $593 \mathrm{~m}^{2}$ will be employed for this measurement.

\section{Beam Exposure and Analysis Scheme}

Two test beam campaigns were held at CERN SPS in 2016 and in 2017. In 2018, a pilot run was conducted aiming at the recording of $10 \%$ of the experimental data. A schematic view of the detector setup is shown in Fig. 3.

The proton beam profile was measured by a silicon pixel telescope. Each emulsion detector module was mounted on a motorized X-Y stage (target mover) to change the position of the module with respect to the proton beam, so to make the detector surface uniformly irradiated at a density of $10^{5}$ tracks $/ \mathrm{cm}^{2}$.

The emulsion detector is both a detector and the data storage media at the same time. The automatic scanning systems read out the track information accumulated in the emulsion films during the exposure,

ISSN 2071-0194. Ukr. J. Phys. 2019. Vol. 64, No. 7 
digitize it, and transfer to the computers for the pattern recognition and track analysis like in case of any electronic detector. The output of the readout is the information on the track segments recorded in the top and bottom layers of a film (microtracks). A segment made by linking the microtracks on two layers in a film is called a basetrack, which is a basic unit of the track information from each emulsion film for the later processing. Each basetrack provides $3 \mathrm{D}$ coordinates $\mathbf{X}=(x, y, z), 3 \mathrm{D}$ vector $\mathbf{V}=\left(\tan \theta_{x}, \tan \theta_{y}, 1\right)$, and $d E / d x$ parameter. The tracks are reconstructed by linking basetracks on different films making use of their position and direction.

The average basetrack efficiency measured with tracks is higher than $95 \%$, which provides the track detection with efficiency $>99 \%$. The reconstructed tracks are then used to find vertices. To provide the efficient detection of small kinks of $D_{s} \rightarrow \tau$ decays, the analysis is performed in two stages: (1) scan the full module by a fast HTS system with relatively coarse angular resolution $(2.5 \mathrm{mrad})$ and detect events that have two decays in a short distance, namely, the decays of $\tau$ and partner charm $\left(D^{ \pm}\right.$and $\left.D^{0}\right) ;(2)$ perform a high-precision measurement around the $\tau$ decay candidates to find $D_{s} \rightarrow \tau$ small kinks. For this, the dedicated stations with a piezo-based $Z$ axis are used providing a reproducibility of a single hit position measurement of $8 \mathrm{~nm}$ and angular measurements of $0.16 \mathrm{mrad}$ (RMS).

Here, the first results acheaved at the first stage of the analysis are presented. Figure 4 shows the distribution of the $Z$ coordinate (along the beam) of the vertices reconstructed in the detector. An enhancement of the vertices in the tungsten target is evident. One can even see the microstructure corresponding to the emulsion layers (of higher density) and plastic bases/spacers. Figure 5 shows the measured multiplicity of charged particles at proton interactions, compared with the prediction by FLUKA. A good agreement of the numbers of observed tracks and expected ones demonstrates a good efficiency of the track reconstruction.

With the data analyzed so far, several events with short-lived particle decays have been already recognized (See an example in Figure 6).

\section{Conclusion and Outlook}

The DsTau experiment is going to study the tau neutrino production following the high-energy pro- ton interactions, which will provide necessary information for future $\nu_{\tau}$ experiments. CERN SPSC recommended approving DsTau in April 2019.

The test of a beam in 2016-2017 and the pilot run in August 2018 were performed, and over 20 million proton interactions in the detector were registered. The emulsion scanning and analysis of these samples are ongoing, which would allow confirming the Ds detection feasibility and the re-evaluation of the $\nu_{\tau}$ cross-section by refining the $\nu_{\tau}$ flux. The full scale study scheduled for the next physics run at CERN SPS in 2021 and 2022. A large amount of the decays of charmed particles is expected to be recorded, as well providing a possibility of interesting by-product results.

1. M. Perl et al. Evidence for anomalous lepton production in $e^{+} e^{-}$annihilation. Phys. Rev. Lett. 35, 1489 (1975).

2. K. Kodama et al. Observation of tau neutrino interactions. Phys. Lett. B 504, 218 (2001).

3. N. Agafonova et al. Discovery of $\tau$ neutrino appearance in the CNGS neutrino beam with the OPERA experiment. Phys. Rev. Lett. 115, 121802 (2015).

4. M.G. Aartsen et al. [IceCube Collaboration]. Measurement of atmospheric tau neutrino appearance with IceCube DeepCore. Phys. Rev. D 99, No. 3, 032007 (2019).

5. K. Kodama et al. [DONuT Collaboration]. Final tauneutrino results from the DONuT experiment. Phys. Rev. $D$ 78, 052002 (2008).

6. J.P. Lees et al. [BABAR Collaboration]. Evidence for an excess of $\bar{B} \rightarrow D^{(*)} \tau^{-} \bar{\nu}_{\tau}$ decays. Phys. Rev. Lett. 109, 101802 (2012).

7. R. Aaij et al. [LHCb Collaboration]. Test of lepton universality using $B^{+} \rightarrow K^{+} l^{+} l^{-}$decays. Phys. Rev. Lett. 113, 151601 (2014).

8. R. Aaij et al. [LHCb Collaboration]. Measurement of the ratio of branching fractions $B\left(\bar{B}^{0} \rightarrow D^{*+} \tau^{-} \bar{\nu}_{\tau}\right) / B\left(\bar{B}^{0} \rightarrow\right.$ $\left.\rightarrow D^{*+} \mu^{-} \bar{\nu}_{\mu}\right)$. Phys. Rev. Lett. 115, No. 11, 111803 (2015).

9. H. Liu, A. Rashed, A. Datta. Probing lepton nonuniversality in tau neutrino scattering. Phys. Rev. D 92, 073016 (2015).

10. Y. Fukuda et al. [Super-Kamiokande Collaboration]. Measurement of a small atmospheric $\nu_{\mu} / \nu_{e}$ ratio. Phys. Let. B 433, Iss. 1, 9 (1998).

11. R. Acciarri et al. [DUNE Collaboration]. Long-Baseline Neutrino Facility (LBNF) and Deep Underground Neutrino Experiment (DUNE). Vol. 1. The LBNF and DUNE Projects, FERMILAB-DESIGN-2016-01 (2016).

12. K. Abe et al. [Hyper-Kamiokande Proto-Collaboration]. Physics potential of a long-baseline neutrino oscillation experiment using a J-PARC neutrino beam and HyperKamiokande. PTEP 2015, 053C02 (2015). 
13. R. Wendell, K. Okumura. Recent progress and future prospects with atmospheric neutrinos. New J. Phys. 17, 025006 (2015).

14. D. Meloni. On the systematic uncertainties in DUNE and their role in New Physics studies. JHEP 1808, 028 (2018). e-Print: arXiv:1805.01747 [hep-ph].

15. J. Blietschau et al. [Gargamelle Collaboration]. Total cross sections for $\nu_{e}$ and $\bar{\nu}_{e}$ interactions and search for neutrino oscillations and decay. Nucl. Phys. B 133, 205 (1978).

16. K.A. Olive et al. [Particle Data Group]. Review of Particle Physics. Chin. Phys. C 38, 090001 (2014).

17. Z. Li et al. [SuperKamiokande Collaboration], Measurement of the tau neutrino cross-section in atmospheric neutrino oscillations with Super-Kamiokande. Phys. Rev. D 98, No. 5, 052006 (2018); arXiv:1711.09436 [hep-ex].

18. N. Agafonova et al. [OPERA Collaboration]. Final results of the OPERA experiment on $\nu_{\tau}$ appearance in the CNGS neutrino beam. Phys. Rev. Lett. 120, No. 21, 211801 (2018). Erratum: [Phys. Rev. Lett. 121, No. 13, 139901 (2018).] [arXiv:1804.04912 [hep-ex]].

19. M. Anelli et al. [SHiP Collaboration]. A facility to search for hidden particles (SHiP) at the CERN SPS, CERNSPSC-2015-016, SPSC-P-350 (2015).

20. M. De Serio [SHiP Collaboration]. Neutrino physics with the SHiP experiment at CERN, PoS EPS-HEP2017 (2017) 101

21. I. Abt et al. [HERA-B Collaboration]. Measurement of $D^{0}$, $D^{+}, D s^{+}$and $D^{*+}$ production in fixed target $920 \mathrm{GeV}$ proton-nucleus collisions. Eur. Phys. J. C 52, 531 (2007).

22. K. Kodama et al. [Fermilab E653 Collaboration]. Charm meson production in $800-\mathrm{GeV} / \mathrm{c}$ proton - emulsion interactions. Phys. Lett. B 263, 573 (1991).

23. R. Ammar et al. [LEBC-MPS Collaboration]. D-Meson Production in $800-\mathrm{GeV} / \mathrm{c}$ pp interactions. Phys. Rev. Lett. 61, 2185 (1988).

24. G.A. Alves et al. [E769 Collaboration]. Feynman-x and transverse momentum dependence of $\mathrm{D}$ meson production in $250 \mathrm{GeV} \mathrm{p}, \mathrm{K}$, and p interactions with nuclei. Phys. Rev. Lett. 77, 2392 (1996).

25. M. Kaya et al. [SELEX Collaboration], Production asymmetry of D(s) from 600-GeV/c Sigma- and pi-beam. Phys. Lett. $B$ 558, 34 (2003).

26. T. Nakano, M. Yoshimoto, R. Komatani. The Newest Technique for Nuclear Emulsion Readout. J. of Society of Photographic Sci. and Techn. of Japan 79, 54 (2016).

27. M. Yoshimoto, T. Nakano, R. Komatani, H. Kawahara. Hyper-track selector nuclear emulsion readout system aimed at scanning an area of one thousand square meters. PTEP 10, 103 (2017).

28. A. Ariga, T. Ariga. Fast $4 \pi$ track reconstruction in nuclear emulsion detectors based on GPU technology. JINST 9, P04002 (2014).

29. T. Sjostrand et al. A brief introduction to PYTHIA 8.1. Comput. Phys. Commun. 178, 852 (2008).

30. N. Agafonova et al. [OPERA Collaboration]. Momentum measurement by the multiple Coulomb scattering method in the OPERA lead emulsion target. New J. Phys. 14, 013026 (2012).

31. T. Nakamura, et al. The OPERA film: New nuclear emulsion for large-scale, high-precision experiments. Nucl. Instrum. Meth. A 556, 80 (2006).

32. S. Aghion et al. Prospects for measuring the gravitational free-fall of antihydrogen with emulsion detectors. JINST 8, P08013 (2013).

33. E. Eskut et al. New results from a search for $\nu_{\mu} \rightarrow \nu_{\tau}$ and $\nu_{e} \rightarrow \nu_{\tau}$ oscillation. Phys. Lett. B 497, 8 (2001).

34. S. Aoki, et al. Experimental proposal, study of tauneutrino production at the CERN-SPS, CERN-SPSC2017-029, SPSC-P-354, arXiv:1708.08700.

35. T.T. Böhlen, F. Cerutti, M.P. W. Chin, A. Fasso, A. Ferrari, P.G. Ortega, A. Mairani, P.R. Sala, G. Smirnov, V. Vlachoudis. The FLUKA code: developments and challenges for high energy and medical applications. Nucl. Data Sheets 1 20, 211 (2014).

Received 08.07.19

Ю. Горнушкін

ДОСЛІДЖЕННЯ ПРОДУКУВАННЯ

ТАУ-НЕЙТРИНО В ПРОТОН-ЯДЕРНІЙ ВЗАЄМОДІЇ

$\mathrm{P}$ е $з$ ю м е

B рамках експерименту DsTau на прискорювачі SPS в ЦЕРНі нами запропоновано незалежний та прямий спосіб дослідження продукування тау-нейтрино в високоенергетичних зіткненнях протонів з ядрами. Зважаючи не те, що основним джерелом нейтрино $є$ розпад Ds-мезонів, в проекті будуть вимірюватись диференційні перерізи цього процесу. Методика експерименту базується на застосуванні емульсійних детекторів для ефективної реєстрації подій розпаду короткоживучих частинок. Нами представлено мотивацію проекту, деталі експериментальної техніки, а також перші результати аналізу даних з перших пробних сеансів, що показали ефективність нашого експерименту. 